\title{
DIFERENCIA EN LAS VARIABLES ASOCIADAS A LA POTENCIA ENTRE JUGADORES UNIVERSITARIOS DE FÚTBOL SOCCER Y FÚTBOL RÁPIDO
}

\section{DIFFERENCE IN THE VARIABLES ASSOCIATED WITHPOWER BETWEEN UNIVERSITY PLAYERS OF SOCCER AND FAST SOCCER}

Hernández Prieto, $\mathrm{YH}^{1}$; Avendaño Coss, $\mathrm{RA}^{2}$; García Oceguera, $\mathrm{D}^{3}$ y Quintin Fernández, ${ }^{4}$

1 Dr. Yuri Hernando Hernández Prieto: Facultad de Ciencias del Deporte. Universidad Autónoma del Estado de Morelos. México. yuri.hernando@uaem.mx

2 Raúl Alfonso Avendaño Coss: Facultad de Ciencias del Deporte. Universidad Autónoma del Estado de Morelos. México. raulcossalfonso@gmail.com

${ }^{3}$ Dulce García Oceguera: Facultad de Ciencias del Deporte. Universidad Autónoma del Estado de Morelos. México.dulgarco@gmail.com

${ }^{4}$ Mtro. Eduardo Quintín Fernández: Facultad de Ciencias del Deporte. Universidad Autónoma del Estado de Morelos. México. eduardo.quintin@uaem.mx

Código UNESCO: 5801 Teoría y Métodos Educativos.

Clasificación Consejo de Europa: 12. Aprendizaje Motor.

Recibido el 26 de febrero de 2018

Aceptado el 12 de junio de 2018

Correspondencia:

Dr. Yuri Hernando Hernández Prieto

yuri.hernando@uaem.mx

Financiación: Prodep

DOI: http://dx.doi.org/10.24310/riccafd.2018.v7i2.5090

\section{RESUMEN}

En el presente estudio, se pretendió comparar los valores de potencia de jugadores de fútbol rápido y fútbol soccer de categoría universitaria. Se reclutó a 10 jugadores de fútbol soccer (22 \pm 2.89 años) grupo FUTSOCC y 10 jugadores de fútbol rápido (21.1 \pm 1.6 años) grupo FUTRAP. Se ejecutó el ejercicio de media sentadilla cargada incrementando el peso en cada serie, se evaluaron las siguientes variables: $1 \mathrm{RM}$, porcentaje de RM con mejor potencia media, potencia media obtenida y el tiempo que tardaban en alcanzar la potencia pico. Se estableció un nivel de significación de $p \leq 0.05$. Los resultados mostraron que el grupo FUTSOCC tuvo mayor 1RM, mientras que el grupo FUTRAP obtuvo su mejor valor de potencia media con porcentajes de $1 \mathrm{RM}$ mayores al igual que sus tiempos de expresión de potencia pico fueron menores en comparación con el 
FUTSOCC. En los valores de potencia media no hubo diferencias significativas. Los resultados nos hacen concluir que los jugadores de fútbol soccer tienen niveles más elevados de fuerza máxima, pero, los jugadores de fútbol rápido aplican su fuerza en menor tiempo lo que lleva a ser más veloces en la ejecución del movimiento.

Palabras claves: potencia, fútbol, fuerza.

\section{ABSTRACT}

The aim of this study was to compare the power values of university indoor soccer and field soccer players. For the purpose of the study, 10 soccer players (22 \pm 2.89 years) FUTSOCC group, and 10 fast soccer players $(21.1 \pm 1.6$ years) FUTRAP group, were recruited. The protocol was based on the execution of the exercise of half squat increasing the weight in each series. The following variables were assessed: 1RM, percentage of $1 \mathrm{RM}$ with better average power, average power obtained and the time it took to reach the peak power. A level of significance of $p \leq 0.05$ was established. The results showed that the FUTSOCC group had higher 1RM, while the FUTRAP group reached higher 1RM percentages at their best average power value and their peak power expression times were lower. In the mean power values there were no significant differences. The results make us conclude that field soccer players have higher levels of maximum strength, but, indoor soccer players are faster in the execution of the movement.

Key words: power, football, strength, performance

\section{INTRODUCCIÓN}

La fuerza es la cualidad física por excelencia, debido a que es la que permite optimizar el rendimiento en acciones explosivas, rápidas y de corta duración. Estas acciones explosivas requieren en gran medida de un equilibrio entre fuerza y velocidad lo que se denomina potencia muscular en los miembros inferiores $^{(1)}$. Al igual para la ejecución de acciones explosivas el ciclo acortamiento-estiramiento (CAE) es un tipo natural de función muscular en donde el músculo es estirado inmediatamente antes de contraerse (2), requiriendo para su correcta puesta en escena, elevados valores de potencia que se manifiesten entre 250 y 300 milisegundos ${ }^{(3)}$. Esta característica coloca a la potencia muscular como un factor fundamental para el adecuado desempeño de atletas en deportes multidireccionales ${ }^{(3)}$, destacándose como un predictor de desempeño en la aceleración ${ }^{(4,5)}$, velocidad máxima ${ }^{(5,6)}$ y en características generales de estas disciplinas ${ }^{(7,8)}$.

Un deporte donde se destaca la necesidad de un alto desempeño en velocidad máxima y en cambios de dirección es el fútbol soccer ${ }^{(9)}$ donde del $1-4 \%$ de la distancia total recorrida durante un partido es en sprints con duraciones de 2-4 segundos ${ }^{(5)}$. El fútbol rápido, debido a que presenta constantes aceleraciones, desaceleraciones (CAE) y cambios de dirección, (aun mas que en el fútbol soccer) la potencia es determinante. Si bien existen parecidos importantes entre ambas disciplinas deportivas, la diferencia más

62 JUGADORES UNIVERSITARIOS DE FÚTBOL SOCCER Y FÚTBOL RÁPIDO 
grande radica en las medidas y perímetros de la cancha, mientras que en fútbol soccer oscila entre los 90-120 m de largo por 65-75 m de ancho, la cancha de fútbol sala (rápido) tiene unas medidas de $65 \mathrm{~m}$ de largo por $30 \mathrm{~m}$ de ancho, lo que resulta en un juego mucho más rápido.

Es evidente que en ambas disciplinas tener altos valores de potencia es vital para el adecuado rendimiento de los atletas, sin embargo las diferencias existentes en las dimensiones del área de juego y por lo tanto el ritmo de los partidos, nos hace pensar que los valores de potencia que se producen en cada disciplina pueden ser distintos debido a las adaptaciones a las que se ve obligado el jugador entorno a las dimensiones del campo y el número de jugadores. A consideración de las condiciones mencionadas anteriormente, el objetivo del estudio es conocer si existen diferencias en los valores de potencia y los tiempos de expresión de la misma, en media sentadilla cargada, que pueden generar los jugadores de fútbol rápido a comparación de los jugadores de fútbol soccer.

\section{MATERIALES Y MÉTODOS}

\section{Participantes}

La muestra objeto del estudio estuvo compuesta por 20 jugadores con una edad media $(21.5 \pm 2.3)$ años, peso $(72.2 \pm 8.1) \mathrm{kg}$, estatura $(170 \pm .05) \mathrm{cm}$, de federados universitarios en el estado de Morelos que compiten en la tercera división de fútbol rápido y fútbol soccer respectivamente. Todos los jugadores tenía una experiencia de práctica en el deporte superior a 5 años. La discriminación de la muestra fue la siguiente: 10 jugadores de fútbol soccer pertenecientes a la tercera división (FUTSOCC) $(71.5 \pm 7.1 \mathrm{~kg} / 168 \pm .05 \mathrm{~cm} /$ $22 \pm 2.89$ años) y 10 jugadores pertenecientes a una selección universitaria de fútbol rápido (FUTRAP) $(72.9 \pm 9.4 \mathrm{~kg} / 171 \pm .05 \mathrm{~cm} / 21.1 \pm 1.6$ años).

\section{Material}

Para el tratamiento de los datos se utilizaron los programas estadísticos EXCEL 365 y SPSS 21.

Para el desarrollo del test de potencia se utilizó peso libre con acabado en caucho marcha (Firesport), 2 barras olímpicas de 2.20 m y $20 \mathrm{~kg}, 2$ discos de $20 \mathrm{~kg}, 2$ discos de $10 \mathrm{~kg}, 2$ discos de $5 \mathrm{~kg}$.

\section{Procedimiento}

Antes del inicio del estudio, se informó a los participantes, de las características y objetivos del mismo, solicitando su aprobación. Cada participante diligenció un documento de consentimiento, informando su participación voluntaria, después se recogieron los datos generales de la muestra, correspondientes al peso talla, y fecha de nacimiento, el grupo fue contactado en el mes de junio, encontrándose en la última fase del periodo 
competitivo y habiendo transcurrido más de la mitad de la temporada. Al inicio del estudio se trabajó una semana de introducción, en dos sesiones de entrenamiento, familiarizándose en los ejercicios con cargas, con los que iba a desarrollar la evaluación.

Se procedió a evaluar a los jugadores en los siguientes test: análisis:

Se desarrolló una evaluación con pesos crecientes, que consto de dos

1- Se evaluó la media sentadilla, en un test con pesos crecientes para:

- Determinar las cargas donde se encuentra el peso óptimo, para el mejor valor de potencia media en cinco repeticiones. Bryzic.

- Encontrar la RM por predicción estadística mediante el índice de

2- Se determinó dentro de las 5 repeticiones con el peso optimo el pico de potencia y acto seguido se determinó el tiempo en el cual se produjo cada pico de potencia.

Antes de cada sesión de evaluación, los sujetos realizaron un calentamiento general, basado en una activación aeróbica, mediante carrera continua durante siete minutos, movilidad articular dinámica durante diez minutos, cinco minutos estiramientos estáticos en las extremidades inferiores (uno de cuádriceps, dos de isquiotibiales, uno de aductores, uno de psoas-iliaco).

Las variables evaluadas fueron:

1. La potencia media generada en cinco repeticiones en media sentadilla.

2. $1 \mathrm{RM}$ por predicción siguiendo el índice de Bryzic

3. El porcentaje del RM con el cual obtuvieron mejor potencia media.

4. Con la carga en la cual se manifestó la mejor potencia media en cinco repeticiones se buscó el promedio de tiempo en milisegundos donde se logró cada pico de potencia entre la segunda a la quinta repetición.

\section{Protocolos de Evaluación}

Se realizó un test incremental, utilizando para la evaluación el encoder lineal Isocontrol 5.2. Se realizó el ejercicio en series de cinco repeticiones. La primera serie se desarrolló, tan solo con la barra, cuyo peso es de $20 \mathrm{~kg}$, después se le sumaron $10 \mathrm{~kg}$ a la barra en cada serie hasta alcanzar los $50 \mathrm{~kg}$, a partir de donde se fueron sumando $5 \mathrm{~kg}$ en cada serie hasta encontrar el mejor valor de potencia.

En todas las series se mantuvo un total de cinco repeticiones y de media, cada sujeto tuvo un tiempo de recuperación, entre series, de cuatro a cinco minutos. El ejercicio se desarrolló en gimnasio, con el peso libre, sin ningún tipo de maquinaria adicional y el valor de potencia media se obtuvo, mediante el software Isocontrol 6.0. Cabe anotar que para el cálculo de potencia en el

64 DIFERENCIA EN LAS VARIABLES ASOCIADAS A LA POTENCIA ENTRE JUGADORES UNIVERSITARIOS DE FÚTBOL SOCCER Y FÚTBOL RÁPIDO 
software, la carga que se introdujo fue la suma de la carga externa más el peso corporal.

Para el segundo punto, relacionado con la obtención del 1RM en el mismo ejercicio, se aplicó el índice de Bryzick 1RM = Peso Levantado/[1,0278-(0,0278 x № de Repeticiones)].

\section{Análisis estadístico}

Para el análisis estadístico se estableció un nivel de significación de $\mathrm{p} \leq 0.05$.

Para determinar la normalidad de la muestra, se utilizó la prueba no paramétrica de Kolmogorov-Smirnov para una muestra. Se calcularon los estadísticos descriptivos (media \pm desviación estándar), de las diferentes variables analizadas para cada uno de los grupos que componen la muestra.

T de Student para muestras independientes: Aplicada entre los grupos a) para comparar los resultados de la evaluación y determinar si existe diferencias significativas a favor de alguno de los grupos.

\section{RESULTADOS}

Tras aplicar la prueba de normalidad, se comprobó que no existen diferencias significativas entre los sujetos de ambos grupos. En cuanto al análisis de la variable Repetición Máxima (RM) los resultados que se muestran en la tabla 1, revelan que el grupo FUTSOCC tiene una media $(111.6 \mathrm{~kg})$ un $13 \%$ mayor respecto al grupo FUTRAP $(98.6 \mathrm{~kg})$.

Tabla 1. Descriptivos de repetición máxima $(\mathrm{RM})$.

\begin{tabular}{|l|l|r|r|r|r|}
\hline & Deporte & $\mathrm{N}$ & Media & \multicolumn{1}{c|}{$\begin{array}{c}\text { Desviación } \\
\text { Estándar }\end{array}$} & $\begin{array}{c}\text { Error estándar } \\
\text { de media }\end{array}$ \\
\hline RM: $\begin{array}{l}\text { Repetición } \\
\text { máxima }(\mathrm{kg})\end{array}$ & FUTSOCC & 10 & 111.6 & 11.20714 & 3.54401 \\
\cline { 2 - 6 } & FUTRAP & 10 & 98.6 & 8.93433 & 2.82528 \\
\hline
\end{tabular}

Al aplicar la prueba de T Student para muestras independientes, el análisis mostró que existe una diferencia significativa entre el resultado del grupo FUTSOCC y FUTRAP $p=0.010$. Ver Tabla 5

En relación a la variable asociada a la carga con la cual se obtiene el mejor valor de potencia media en cinco repeticiones, se determinó que el porcentaje de RM en el cual se logró la mejor potencia media para cada grupo según los 
estadísticos descriptivos es el $50 \%$ de la RM para el FUTSCC y del $62 \%$ de la RM para el FUTRAP como queda reflejado en la siguiente tabla.

Tabla 2. Descriptivo \%RM con mejor potencia media.

\begin{tabular}{|c|c|c|c|c|c|}
\hline & Deporte & $N$ & Media & $\begin{array}{l}\text { Desviación } \\
\text { Estándar }\end{array}$ & $\begin{array}{c}\text { Error estándar } \\
\text { de media }\end{array}$ \\
\hline \multirow{2}{*}{$\begin{array}{l}\text { \%RM: } \\
\text { Porcentaje de } \\
\text { la repetición } \\
\text { máxima con } \\
\text { mejor potencia } \\
\text { media (\%) }\end{array}$} & FUTSOCC & 10 & 50.2 & 8.56089 & 2.70719 \\
\hline & FUTRAP & 10 & 61.8 & 9.95322 & 3.14749 \\
\hline
\end{tabular}

Al aplicar la prueba de T Student para muestras independientes, el análisis evidenció que existe una diferencia significativa entre el resultado del grupo FUTSOCC y FUTRAP $\mathrm{p}=0.012$ (Tabla 5).

Continuando con el estudio en relación con la potencia media generada los estadísticos descriptivos, revelan que el grupo FUTSOCC tiene una media (1059W) 9\% mayor al grupo FUTRAP (964.1W) como se puede observar en la Tabla 3.

Tabla 3. Descriptivos de la Potencia Media Generada.

\begin{tabular}{|l|l|r|r|r|r|}
\hline & Deporte & $\mathrm{N}$ & Media & \multicolumn{1}{c|}{$\begin{array}{c}\text { Desviación } \\
\text { Estándar }\end{array}$} & $\begin{array}{l}\text { Error estándar } \\
\text { de media }\end{array}$ \\
\hline $\begin{array}{l}\text { Pmedia: } \\
\text { Potencia } \\
\text { Media (W) }\end{array}$ & FUTSOCC & 10 & 1059 & 279.66965 & 88.43931 \\
\cline { 2 - 6 } & FUTRAP & 10 & 964.1 & 198.13601 & 62.65611 \\
\hline
\end{tabular}

Al aplicar la prueba de T Student para muestras independientes, no se detectaron diferencias significativas entre el resultado del grupo FUTSOCC y FUTRAP $p=0.393$. Ver Tabla 5

Finalmente en relación al promedio de tiempo en el que se alcanzan los picos de potencia (TiPpico), los estadísticos descriptivos, revelan que el grupo FUTSOCC tiene una media (559.2 ms) 27.1\% mayor al grupo FUTRAP (407.8 $\mathrm{ms})$. Tal y como se muestra seguidamente. 
Tabla 4. Descriptivos de promedio de tiempo para pico de potencia.

\begin{tabular}{|l|l|r|r|r|r|}
\hline & Deporte & $\mathrm{N}$ & Media & \multicolumn{1}{c|}{$\begin{array}{c}\text { Desviación } \\
\text { Estándar }\end{array}$} & $\begin{array}{l}\text { Error estándar } \\
\text { de media }\end{array}$ \\
\hline $\begin{array}{l}\text { TiPpico: } \\
\begin{array}{l}\text { Promedio de } \\
\text { tiempo para } \\
\text { potencia pico } \\
(\mathrm{ms})\end{array}\end{array}$ & FUTSOCC & 10 & 559.2 & 171.18723 & 54.13415 \\
\cline { 2 - 6 } & FUTRAP & 10 & 407.8 & 65.18146 & 20.61219 \\
\hline
\end{tabular}

Al aplicar la prueba de T Student para muestras independientes, el análisis expuso que existe una diferencia significativa entre el resultado del grupo FUTSOCC y FUTRAP $p=0.018$ (Tabla 5).

Tabla 5. Tabla de Significancia al aplicar la Prueba T Student para muestras independientes.

\begin{tabular}{|c|c|c|c|c|c|c|}
\hline \multicolumn{7}{|c|}{ T Student de Muestras Independientes } \\
\hline & & \multicolumn{2}{|c|}{$\begin{array}{l}\text { Prueva de Levene para } \\
\text { Igualdad de Varianzas }\end{array}$} & \multicolumn{3}{|c|}{ Prueba T para Igualdad de Medias } \\
\hline & & \begin{tabular}{l|l}
$\mathrm{F}$ \\
\end{tabular} & Sig. & $\mathrm{t}$ & $\mathrm{df}$ & Sig. (bilateral) \\
\hline \multirow[t]{2}{*}{$\mathrm{RM}(\mathrm{Kg})$} & $\begin{array}{l}\text { Se han asumido varianzas } \\
\text { iguales }\end{array}$ & \multirow[t]{2}{*}{.789} & \multirow[t]{2}{*}{.386} & 2.868 & 18 & .010 \\
\hline & $\begin{array}{l}\text { No se ha asumido } \\
\text { varianzas iguales }\end{array}$ & & & 2.868 & 17.148 & .011 \\
\hline \multirow[t]{2}{*}{$\begin{array}{l}\text { \% RM con mejor potencia } \\
\text { media }\end{array}$} & $\begin{array}{l}\text { Se han asumido varianzas } \\
\text { iguales }\end{array}$ & \multirow[t]{2}{*}{.027} & \multirow[t]{2}{*}{.872} & -2.794 & 18 & .012 \\
\hline & $\begin{array}{l}\text { No se ha asumido } \\
\text { varianzas iguales }\end{array}$ & & & -2.794 & 17.606 & .012 \\
\hline \multirow[t]{2}{*}{ Potencia Media (W) } & $\begin{array}{l}\text { Se han asumido varianzas } \\
\text { iguales }\end{array}$ & \multirow[t]{2}{*}{.592} & \multirow[t]{2}{*}{.451} & .876 & 18 & .393 \\
\hline & $\begin{array}{l}\text { No se ha asumido } \\
\text { varianzas iguales }\end{array}$ & & & .876 & 16.217 & .394 \\
\hline \multirow[t]{2}{*}{$\begin{array}{l}\text { Promedio de tiempo para } \\
\text { la potencia pico (ms) }\end{array}$} & $\begin{array}{l}\text { Se han asumido varianzas } \\
\text { iguales }\end{array}$ & \multirow[t]{2}{*}{4.613} & \multirow[t]{2}{*}{.046} & 2.614 & 18 & .018 \\
\hline & $\begin{array}{l}\text { No se ha asumido } \\
\text { varianzas iguales }\end{array}$ & & & 2.614 & 11.556 & .023 \\
\hline
\end{tabular}

\section{DISCUSIÓN}

Este estudio refleja que existen diferencias significativas entre jugadores fútbol soccer y fútbol rápido de nivel universitario en dos de las tres variables estudiadas fuerza máxima (RM), porcentaje del RM con el que consiguen el mejor valor de potencia media y el tiempo que tardan en alcanzar su mejor expresión de potencia con ese porcentaje de carga. Los resultados son congruentes con la hipótesis para este estudio, la cual mencionaba que existirían 
diferencias en la variables asociadas a la potencia, en media sentadilla cargada, entre los grupos de estudio debido a las características propias de cada juego.

Los resultados demostraron que los jugadores de fútbol soccer tienen niveles de fuerza máxima mayores que los jugadores de fútbol rápido en el ejercicio de media sentadilla en un nivel de significación de $p=0.010$. Por el contrario cuando analizamos el porcentaje del RM donde alcanzan su mejor valor de potencia media (\%RM) el grupo de FUTRAP fue significativamente mejor ( $p$ $=0.012$ ) y logró obtener mejores valores en esta variable al compararlo con el grupo del grupo de FUTSOCC. La misma situación se presentó en la variable asociada al tiempo que se tarda en alcanzar el pico de potencia con la carga con la cual expresa su mejor potencia media (TiPpico), siendo significativamente mejor el grupo de FUTRAP $(p=0.018)$ reflejando que necesitan menos tiempo para llegar a su picos de potencia en el análisis de la segunda a la quinta repetición en comparación con el grupo FUTSOCC.

Para explicar las diferencias en los niveles de fuerza máxima podemos suponer que el grupo FUTSOCC tiene mayor experiencia, dedican más tiempo al entrenamiento fuerza o hacen un mayor enfoque en el trabajo de fuerza máxima que el grupo FUTRAP; pero si consideramos que jugadores de fútbol soccer la fuerza máxima ha mostrado una correlación fuerte con los saltos y sprints cortos lineales ${ }^{(5,10)}$, y a su vez sea citado en la literatura como factor importante para mejorar los niveles de potencia en movimientos explosivos (con un tiempo de ejecución menor a 300 milisegundos) (10), atendiendo a estos autores se puede inferir que el futbolista de Soccer en competición debido a la característica del campo y a la dinámica del juego se ve expuesto a carreras lineales cortas (entre 12 y $20 \mathrm{~m}$ ) y saltos continuos situación que puede favorecer niveles más importantes de fuerza máxima al compararlo con los futbolistas de rápido y argumentar que la ejecución de estos gestos explosivos desarrollados constantemente tiene la trasferencia directa en el ejercicio de media sentadilla; Sin embargo en contraposición Requena, B y col $(2009)^{(11)}$ solo encontró una correlación moderada entre el ejercicio de media sentadilla cuando este se ejecutaba con un $75-115 \%$ del peso corporal y el desempeño funcional de jugadores de soccer en acciones explosivas como saltos y sprints; de modo que según este autor la fuerza máxima no tiene relación con las adaptaciones obtenidas por las características de la cancha y los gestos propios de cada una de las disciplinas, sino por el entrenamiento de fuerza específico realizado por cada grupo.

Aunque se presento está diferencia de RM a favor del grupo de FUTSOCC, los datos de \%RM fueron mejores para el grupo FUTRAP $51 \%$ y $60 \%$ respectivamente. Los resultados de \%RM coinciden con los datos de otros estudios que mencionan que los mejores valores medios y pico de potencia en jugadores de rugby se encuentran entre el $40-60 \% R M{ }^{(13)}$ y en jugadores juveniles de fútbol soccer cerca al 50\%RM ${ }^{(12)}$, sin embargo González Rave, JM; Navarro Valdivieso, F; Delgado Fernández, M; García García JM. (2010)(14) sostienen que en las disciplinas donde el empleo de la fuerza máxima es 
relevante para la actividad (Judo, Lucha ... etc), el porcentaje del RM donde se manifiesta el mejor valor de potencia es mayor, en nuestro estudio esta situación no se dio a pesar de que el fútbol Soccer tiene requerimientos en cuanto a la fuerza máxima mayores que el fútbol rápido, sin ser en ninguna de las dos disciplinas la fuerza máxima una variable determinante en el rendimiento como si lo puede ser la potencia. Es posible que estos resultados estén asociados a la falta de experiencia en el manejo de la carga.

En consonancia según marca la teoría el grupo de FUTSOCC debería haber generado mayor potencia media en cinco repeticiones y con un \%RM mayor al del grupo de FUTRAP, ya que manifiesta mayor fuerza máxima, situación que no se dio al no existir diferencias significativas $(p=0.393)$ en la potencia media, y por el contrario el grupo FUTRAP encontró dicha potencia con un \%RM significativamente mayor. Así pues los resultados marcan que cada grupo compensó sus deficiencias de tal manera que los jugadores de FUTSOCC tuvieron que agregar velocidad a sus movimiento para obtener sus valores de potencia y el grupo de FUTRAP generó su potencia manteniendo su velocidad y aplicando mayor fuerza ya que para obtener dichos valores debieron movilizar un \%RM mayor en comparación con el FUTSOCC.

A la luz de los resultados es evidente que el grupo FUTRAP genera potencia de una manera mas equilibrada entre el componente velocidad y el componente fuerza y esto es claramente reflejado al analizar la variables \%RM y los tiempos en los cuales se consigue el pico de potencia con la carga asignada ya que en esta última, el grupo de FUTRAP también logró un mejor resultado que el grupo de FUTSOCC (407 y 559 milisegundos de media entre los picos de potencia entre la segunda y la quinta repetición respectivamente), de tal manera que el grupo FUTRAP alcanza mayores \%RM en menor tiempo, lo que lo convierte en mas eficiente, al compararlo con los jugadores de FUTSOCC que mueven \%RM menores en mas tiempo.

Al analizar el resultado de las cuatro variables, observamos que en la Pmedia al no presentarse diferencias significativas entre ambos grupos se puede inferir que la velocidad de movimiento de la barra durante el ejercicio, fue un factor determinante para la potencia generada en el caso del grupo FUTRAP y la fuerza máxima para el grupo FUTSOCC, atendiendo a Hori N. y col. $(2005)^{(16)}$, se podría asumir que el grupo FUTSOCC necesita mayor entrenamiento de fuerza velocidad.

Para defender este argumento se debe considerar que a pesar de que el grupo FUTRAP tuvo mejor resultado en \%RM en el cual expreso su mejor valor de potencia media, en relación con el grupo FUTSOCC que presento un menor \%RM para generar dicha potencia, el grupo FUTSOCC cargaba un peso mayor a causa de que el RM era más elevado, dando como resultado que los valores de potencia media en watts fueran similares en ambos grupos inclusive con las diferencias en el TiPpico y en el \%RM a favor del grupo FUTSOCC . 
En este punto para explicar estas diferencias en la velocidad de movimiento, podríamos sugerir que las expresiones de la fuerza-potencia de cada grupo si se ven altamente afectadas por las dimensiones de las canchas, número de jugadores, y la dinámica propia de cada juego donde, en fútbol rápido al ser un espacio más reducido y con menos jugadores en cancha, el ritmo de juego es más rápido e intenso y las acciones de ataque defensa son mas continuas que en el fútbol soccer (15) lo que como es lógico provoca constantes cambios de dirección y un muy elevado número de sprints mas cortos en tiempo y distancia a los exigidos en el fútbol soccer donde a raíz de sus características propias, las magnitudes y los tiempos de aplicación de la fuerza son distintos (mas elevados) a pesar que los movimientos sean los mismos (cambios de dirección, sprints y saltos)

Cabe anotar que cuando hablamos de alta intensidad de juego en el futbol, hablamos de un tema de aceleraciones, desaceleraciones y cambios de dirección muy intensos, acciones que se ven claramente condicionadas por las dimensiones del campo, el número de jugadores y las dinámicas propias de cada deporte, así los dos se llamen fútbol y tengan un gran número de similitudes en los principios del juego ${ }^{(14)}$.

Tomando en cuenta la posición de Hori N. y col. (2005) ${ }^{(16)}$ que explica que son distintas las cualidades para la expresión de la potencia y que por lo tanto cada una se debe de trabajar de forma específica, es posible pensar que en fútbol rápido la fuerza velocidad sea mas solicitada por la forma en la que se desenvuelven los encuentros, contrastando con el fútbol soccer donde puede la fuerza máxima sí sea más determinante al compararlo con el fútbol rápido.

Independientemente de que el grupo FUTRAP tuviera mejor resultado en el TiPpico, los valores en ambos grupos son muy elevados, ya que según Hernández Prieto (2012) ${ }^{(17)}$ los tiempos de ejecución de los gestos explosivos como aceleraciones, desaceleración, saltos y golpeos están alrededor de los 200-250 milisegundos y sugiere que los ejercicios a realizar a modo de entrenamiento deben de estar cerca de esos valores de tiempo.

Es importante mencionar que estos resultados están condicionados tanto por las características propias del deporte como por el entrenamiento de fuerza que llevan en ambos grupos, es determinante resaltar que un deporte colectivo un entrenamiento de fuerza que no este orientado a la potencia si no a la fuerza pura o fuerza máxima es susceptible a no ajustar las cargas de entrenamiento a los tiempos efectivos de ejecución de dicha fuerza y por lo tanto toda fuerza expresada por encima de 250 milisegundos será eventualmente desaprovechada en deportes como el fútbol soccer y el fútbol rápido ya que de nada le valdrá al deportista ser muy fuerte si no tiempo para expresar su fuerza.

Cabe anotar que la mayoría de los estudios que se utilizaron como apoyo y referencia son enfocados en fútbol soccer, y fútbol sala, esto es debido a que, a nuestro conocimiento, es escasa la información que aborde al fútbol rápido, por lo que los datos citados pueden no ser los más acordes para esa disciplina

70

DIFERENCIA EN LAS VARIABLES ASOCIADAS A LA POTENCIA ENTRE JUGADORES UNIVERSITARIOS DE FÚTBOL SOCCER Y FÚTBOL RÁPIDO 
y dada la relevancia del tema se sugiere en el futuro desarrollar estudios de dinámica de juego en el fútbol rápido donde se midan tiempos de carrera a diferentes velocidades así como aceleraciones, desaceleraciones y cambios de dirección en competición.

\section{CONCLUSIONES}

Los jugadores de fútbol rápido generan potencia desde un mayor equilibrio entre fuerza y velocidad, mientras que los jugadores de fútbol soccer generan potencia prevaleciendo el componente fuerza sobre el componente velocidad.

Las adaptaciones propias y especificas derivadas de cada juego condicionan el rendimiento en fuerza-potencia en jugadores de fútbol soccer y fútbol rápido.

\section{REFERENCIAS}

1. Hernández, Y.H. \& García JM. Efectos de un entrenamiento específico de potencia aplicado a futbolistas juveniles para la mejora de la velocidad lineal. Mot Eur $\mathrm{J}$ Hum Mov. 2012;28:125-44.

2. Flanagan EP, Comyns TM. The Use of Contact Time and the Reactive Strength Index to Optimize Fast Stretch-Shortening Cycle Training. Strength Cond J. 2008;30(5):32-8.

3. M'Cormick BT, Hannon JC, Hicks-little C, Newton M, Shultz B. The Relationship between Change of Direction Speed in the Frontal Plane, Power, Reactive Strength, and Strength. Int J Exerc Sci. 2014;7(4):260-70. Available from:http://www.researchgate.net/profile/Brian_Mccormick3/publication/266855838_Th e_Relationship_between_Change_of_Direction_Speed_in_the_Frontal_Plane_Power_ React ive_Strength_and_Strength/links/543df2f80cf240f04d10ce0d.pdf.

4. Lockie RG, Murphy AJ, Knight TJ, Janse de Jonge X. Factors that differenciate acceleration ability in field sport athletes. J Strength Cond Res. 2011;25(10):2704-14.

5. Chelly M, Chérif N, Amar M, Hermassi S, Fathloun M. Relationship of peak leg power, 1 maximal repetition half back squat, leg muscle volume to $5 \mathrm{~m}$ sprint performance of junior soccer players. J Strength Cond Res. 2010;24(1):266-71.

6. Lockie RG, Murphy AJ, Schultz AB, Knight TJ, Janse de Jonge X. The effects of different speed training protocols on sprint acceleration kinematics and muscle strength and power in field sport athletes. J Strength Cond Res. 2012;26(6):1539-50.

7. Kruger K, Pilat C, Uckert K, Frech T, Mooren FC. Physical Performance Profile Of Handball Players Is Related To Playing Position And Playing Class. J Strength Cond Res. 2014;28(1):117-25.

8. Wagner H, Finkenzeller T, Würth S, Duvillard SP Von. Individual and Team Performance in Team-Handball: A Review. J Sport Med. 2014;13:808-16. Available from:http://web.b.ebscohost.com.www.dbproxy.hu.nl/ehost/pdfviewer/pdfviewer?sid=d7 381e 80-db05-40e3-b396-91ad636b8905@sessionmgr110\&vid=21\&hid=128.

9. Souhaiel, Mohamed; Fathloun, Mourad; Cherif N. Effects of a back squat training program on leg power, jump, and sprint performances in junior soccer players. J Strength Cond Res. 2009;23(8):2241-9.

10. Styles WJ, Matthews MJ, Comfort P. Effects of Strength Training On Squat And Sprint Performance in Soccer Players. J Strength Cond Res. 2016;30(6):1534-9. 
11. Requena, B., González, J., De Villareal, S., Ereline, J., García, I., Gapeyeva, H., and Pääsuke, M. (2009). Functional performance, maximal strength, and power characteristics in isometric and dynamic actions of lower extremities in soccer players. $J$ Strength Cond Res, 23(5),1391-401.

12. Sleivert $G$, Taingahue $M$. The relationship between maximal jump-squat power and sprint acceleration in athletes. Eur J Appl Physiol. 2004;91(1):46-52.

13. Hernández P. YH y García G. JM. Efectos de un entrenamiento de fuerza en futbolistas juveniles españoles, con la carga donde manifiestan el mejor valor de potencia en el ejercicio de salto cargado. Revista Iberoamericana de Ciencias de la Actividad Física y el Deporte. 2014;3(2):9-17.

14. González Rave, JM; Navarro Valdivieso, F; Delgado Fernández, M; García García JM. Fundamentos del Entrenamiento Derportivo. Ed.Wanceulen. Sevilla; 2010.

15. Emery CA, Meeuwisse WH. Risk factors for injury in indoor compared with outdoor adolescent soccer. Am J Sports Med. 2006;34(10):1636-42.

16. Hori N, Newton RU, Nosaka K, Stone MH. Weightlifting Exercises Enhance Athletic Performance That Requires High-Load Speed Strength. 2005;27(4):50-5.

17. Hernández YH, García JM. Efectos de un entrenamiento específico de potencia, aplicado a futbolistas juveniles para la mejora de la velocidad y el salto. Motricidad European Jornal of Human. 2012; 28:125-144.

Referencias totales citadas: 17

Referencias citadas correspondientes a la Rev Ib CC Act Fis Dep: 1 